\title{
Non linear evolution: revisiting the solution in the saturation region
}

\author{
Carlos Contreras, ${ }^{a}$ Eugene Levin ${ }^{a, b}$ and Rodrigo Meneses ${ }^{c}$ \\ ${ }^{a}$ Departamento de Física, Universidad Técnica Federico Santa María and \\ Centro Científico-Tecnológico de Valparaíso, Avda. España 1680, Casilla 110-V, Valparaíso, Chile \\ ${ }^{b}$ Department of Particle Physics, School of Physics and Astronomy, \\ Tel Aviv University, Tel Aviv, 69978, Israel \\ ${ }^{c}$ Escuela de Ingeniería Civil, Facultad de Ingeniería, Universidad de Valparaíso, \\ Avda Errazuriz 1834, Valparaíso, Chile \\ E-mail: carlos.contreras@usm.cl, leving@post.tau.ac.il, \\ rodrigo.meneses@uv.cl
}

ABSTRACT: In this paper we revisit the problem of the solution to Balitsky-Kovchegov equation deeply in the saturation domain. We find that solution has the form given in ref. [23] but it depends on variable $\bar{z}=\ln \left(x^{2} Q_{s}^{2}\right)+$ Const and the value of Const is calculated in this paper. We propose the solution for full BFKL kernel at large $\bar{z}$ in the entire kinematic region that satisfies the McLerran-Venugopalan-type [3-7] initial condition.

KEYwORDS: Resummation, QCD

ARXIV EPRINT: 1406.1212 


\section{Contents}

1 Introduction 1

2 Equation and solution in the momentum space 3

2.1 Equation and geometric scaling solution 3

2.2 General solution and initial condition at $Y=Y_{A} \quad 6$

3 Matching two solutions: at small $\tilde{z}$ and at large $\tilde{z} \quad 10$

$\begin{array}{lll}3.1 & \text { Corrections at large } \tilde{z} & 10\end{array}$

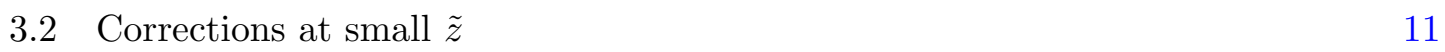

$\begin{array}{lll}3.3 & \text { Matching procedure } & 12\end{array}$

4 Conclusions $\quad 14$

\section{Introduction}

High energy QCD has reached a mature stage [1-10] and has become the common language to discuss high energy scattering where the dense system of partons (quarks and gluons) is produced. The most theoretical progress has been reached in the description of dilute-dense scattering. The deep inelastic scattering of electron is well known example of such process. For these processes the non-linear equations that govern such processes, have been derived and discussed in details [11-21]. The extended phenomenology has been developed based on these equations ${ }^{1}$ which describes the main features of the high energy scattering. For phenomenology the numerical solution to the non-linear equations have been used but it is important to mention that in two limited cases: deeply in the saturation region [23] and in the vicinity of the saturation scale [24-26]; the analytical solutions have been suggested (see ref. [27] where the procedure to incorporate these analytical solutions are suggested that leads to successful description of HERA data).

In this short paper we re-visit the solution deeply in the saturation region [23]. We have two motivations for this. First, in the semi-classical approach [28] we obtain a different solution with the geometric scaling behaviour [29-34] than in ref. [23]. Second, the solution for heavy ions has not been found for the general BFKL kernel $[39,40]$ in spite of several attempts to find it (see refs. [35, 36]).

We start by recalling the derivation of ref. [23]. The non-linear Baslitsky-Kovchegov equation [11-13] takes the form

$$
\frac{\partial N_{01}}{\partial Y}=\bar{\alpha}_{S} \int \frac{d^{2} x_{02}}{2 \pi} \frac{x_{01}^{2}}{x_{02}^{2} x_{12}^{2}}\left\{N_{02}+N_{12}-N_{02} N_{12}-N_{01}\right\}
$$

\footnotetext{
${ }^{1}$ We refer the recent review (see ref. [22]) which, in our opinion, gives both: the up-to-date status report on the theoretical development and the discussion of the phenomenological description of the experimental data in CGC/saturation approach.
} 
where $N_{i k}=N\left(Y, x_{i k}, b\right)$ and $\bar{\alpha}_{S}=\alpha_{S} N_{c} / \pi$. In eq. (1.1) we assume that $b \gg x_{i k}$. Introducing $N_{i k}=1-\Delta_{i k}$ we obtain the following equation for $\Delta_{i k}$ :

$$
\frac{\partial \Delta_{01}}{\partial Y}=\bar{\alpha}_{S} \int \frac{d^{2} x_{02}}{2 \pi} \frac{x_{01}^{2}}{x_{02}^{2} x_{12}^{2}}\left\{\Delta_{02} \Delta_{12}-\Delta_{01}\right\}
$$

Deeply in the saturation region $x_{01}^{2} Q_{s}^{2}(Y, b) \gg 1$ where $Q_{s}^{2}(Y, b)$ is the new scale: saturation momentum. It is equal to (see refs. [1, 24, 25, 37, 38])

In eq. (1.3)

$$
Q_{s}^{2}(Y, b)=Q_{s}^{2}(Y=0, b) e^{\bar{\alpha}_{S} \kappa Y} \quad \text { with } \quad \kappa=\frac{\chi\left(\gamma_{c r}\right)}{1-\gamma_{c r}}
$$

$$
\frac{\chi\left(\gamma_{c r}\right)}{1-\gamma_{c r}}=-\frac{d \chi\left(\gamma_{c r}\right)}{d \gamma_{c r}} \quad \text { and } \quad \chi(\gamma)=2 \psi(1)-\psi(\gamma)-\psi(1-\gamma)
$$

$\chi(\gamma)$ is the kernel of the BFKL linear equation [39, 40] where $\psi(\gamma)=d \ln \Gamma(\gamma) / d \gamma$ is the Euler psi-function (see formula 8.36 of ref. [42]).

Assuming that both $x_{12}$ and $x_{02}$ are in the saturation region, i.e. $x_{12}^{2} Q_{s}^{2}(Y, b)>1$ and $x_{02}^{2} Q_{s}^{2}(Y, b)>1$ we can consider that $\Delta_{i k} \ll 1$ and neglect the term proportional to $\Delta_{02} \Delta_{12}$ in comparison with $\Delta_{01}$. Resulting equation takes the form

$$
\begin{aligned}
\frac{\partial \Delta_{01}}{\partial Y} & =-\bar{\alpha}_{S} \Delta_{01} \int \frac{d^{2} x_{02}}{2 \pi} \frac{x_{01}^{2}}{x_{02}^{2} x_{12}^{2}} \\
& =-\frac{\bar{\alpha}_{S}}{2 \pi} \Delta_{01}\{\underbrace{\pi \int_{1 / Q_{s}^{2}}^{x_{01}^{2}} \frac{d x_{02}^{2}}{x_{02}^{2}}}_{x_{02} \ll x_{01}}+\underbrace{\pi \iint_{1 / Q_{s}^{2}}^{x_{01}^{2}} \frac{d x_{12}^{2}}{x_{12}^{2}}}_{x_{12} \ll x_{01}}\}=-\bar{\alpha}_{S} z \Delta_{01}
\end{aligned}
$$

where we introduce a new variable

$$
z=\ln \left(x_{01}^{2} Q_{s}^{2}(Y, b)\right)=\bar{\alpha}_{S} \kappa Y+\xi
$$

with $\xi=\ln \left(x_{01}^{2} Q_{s}^{2}(Y=0, b)\right)$.

One can see that the solution to eq. (1.5) is

$$
\Delta_{01}=\text { Const } \exp \left(-\frac{z^{2}}{2 \kappa}\right)
$$

It should be stressed that this solution shows the geometric scaling behaviour [29-34] being function of only one variable: $z$.

This derivation shows two problems that have been mentioned above: we need to assume that the main contribution in eq. (1.5) stems from the saturation region; and the answer has a geometric scaling behaviour that contradicts the initial condition for the DIS with nuclei.

Indeed, at $Y=Y_{A}$ for DIS with nuclei we have McLerran-Venugopalan formula for the imaginary part of the dipole-nucleus amplitude, which takes the following form $[3-7,10]$ (see figure 1)

$$
N\left(x^{2}, Y=Y_{A}\right)=1-\exp \left(-\frac{1}{4} x^{2} Q_{s}^{2}\left(Y=Y_{A}, b\right) \ln \left(\frac{1}{x \Lambda}\right)\right)
$$




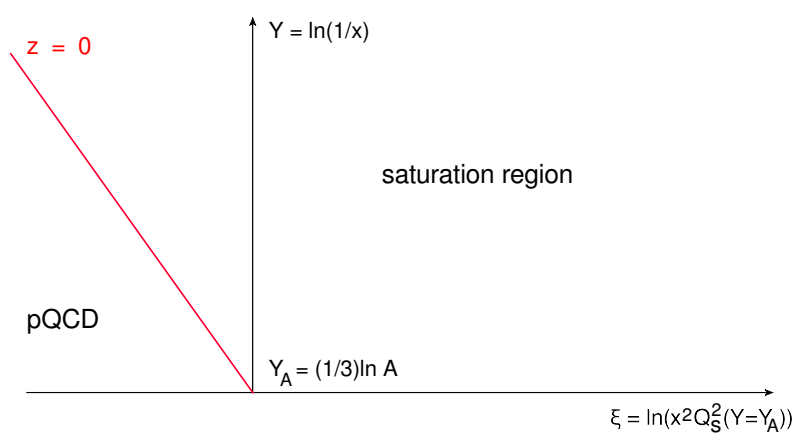

Figure 1. Saturation region of QCD. Red line shows the saturation boundary $(\mathrm{z}=0)$.

One can see that eq. (1.8) does not reproduce the solution of eq. (1.7) at $Y=Y_{A}$. Comparing eq. (1.8) and eq. (1.7) we see that the geometric scaling behaviour cannot be correct in the entire saturation region.

\section{Equation and solution in the momentum space}

\subsection{Equation and geometric scaling solution}

We re-write the Balitsky-Kovchegov equation of eq. (1.1) in the momentum space introducing

$$
N\left(x^{2}, b ; Y\right)=x^{2} \int \frac{d^{2} k_{\perp}}{2 \pi} e^{i \vec{k}_{\perp} \cdot \vec{x}} \widetilde{N}\left(k_{\perp}, b ; Y\right)
$$

It takes the form $[1,41]$

$$
\frac{\partial \widetilde{N}\left(k_{\perp}, b ; Y\right)}{\partial Y}=\bar{\alpha}_{S}\left\{\chi\left(-\frac{\partial}{\partial \tilde{\xi}}\right) \widetilde{N}\left(k_{\perp}, b ; Y\right)-\widetilde{N}^{2}\left(k_{\perp}, b ; Y\right)\right\}
$$

where

$$
\tilde{\xi}=\ln \left(k_{\perp}^{2} / Q_{s}^{2}\left(Y=Y_{A}, b\right)\right) \quad \text { and } \quad \tilde{z}=\bar{\alpha}_{S} \kappa\left(Y-Y_{A}\right)-\tilde{\xi}=\ln \left(Q_{s}^{2}(Y, b) / k_{\perp}^{2}\right)
$$

The advantage of the non-linear equation in eq. (2.2) that the non-linear term depends only on external variable and does not contain the integration over momenta. The BFKL kernel: $\chi\left(-\frac{\partial}{\partial \tilde{\xi}}\right)$, can be written as the series over positive powers of $\partial / \partial \tilde{\xi}$ except of the first term

$$
\frac{1}{\gamma} \widetilde{N}\left(k_{\perp}, b ; Y\right) \rightarrow \int^{k_{\perp}^{2}} \frac{d k_{\perp}^{\prime 2}}{k_{\perp}^{\prime 2}} \widetilde{N}\left(k_{\perp}^{\prime}, b ; Y\right)
$$

Differentiating eq. (2.2) over $\tilde{\xi}$ one can see that it can be re-written as

$$
\begin{aligned}
& \frac{\partial \widetilde{N}_{\tilde{\xi}}^{\prime}\left(k_{\perp}, b ; Y\right)}{\partial Y} \\
= & \bar{\alpha}_{S}\left\{\left(\chi(\gamma)-\frac{1}{\gamma}\right) \widetilde{N}_{\tilde{\xi}}^{\prime}\left(k_{\perp}, b ; Y\right)+\widetilde{N}\left(k_{\perp}, b ; Y\right)-2 \widetilde{N}_{\tilde{\xi}}^{\prime}\left(k_{\perp}, b ; Y\right) \widetilde{N}\left(k_{\perp}, b ; Y\right)\right\}
\end{aligned}
$$

where $\gamma=-\frac{\partial}{\partial \tilde{\xi}}$. 
Introducing the variable $\tilde{z}$ instead of $\tilde{\xi}$ and the new function $M$ as

$$
\widetilde{N}_{\tilde{z}}^{\prime}(\tilde{z}, b ; Y)=\frac{1}{2}+M(\tilde{z}, b ; Y) \quad \text { or } \quad \tilde{N}(\tilde{z}, b ; Y)=\frac{1}{2} \tilde{z}+\int_{0}^{\tilde{z}} d \tilde{z}^{\prime} M\left(\tilde{z}^{\prime}, b ; Y\right)
$$

we can re-write eq. (2.5) in the form

$$
\begin{aligned}
& \bar{\alpha}_{S} \kappa \frac{\partial M(\tilde{z}, b ; \tilde{Y})}{\partial \tilde{z}}+\bar{\alpha}_{S} \frac{\partial M(\tilde{z}, b ; \tilde{Y})}{\partial \tilde{Y}} \\
= & \bar{\alpha}_{S}\left\{\left(\chi(\gamma)-\frac{1}{\gamma}\right) M(\tilde{z}, b ; \tilde{Y})-\tilde{z} M(\tilde{z}, b ; \tilde{Y})-M(\tilde{z}, b ; \tilde{Y}) \int_{0}^{\tilde{z}} d \tilde{z}^{\prime} M\left(\tilde{z}^{\prime}, b ; \tilde{Y}\right)\right\}
\end{aligned}
$$

with $\gamma=\frac{\partial}{\partial \tilde{z}}$ and $\tilde{Y}=\bar{\alpha}_{S} Y$.

We can re-write eq. (2.7) excluding dependence on $\bar{\alpha}_{S}$ and it takes the form

$$
\begin{aligned}
& \kappa \frac{\partial M(\tilde{z}, b ; \tilde{Y})}{\partial \tilde{z}}+\frac{\partial M(\tilde{z}, b ; \tilde{Y})}{\partial \tilde{Y}} \\
= & \left(\chi(\gamma)-\frac{1}{\gamma}\right) M(\tilde{z}, b ; \tilde{Y})-\tilde{z} M(\tilde{z}, b ; \tilde{Y})-M(\tilde{z}, b ; \tilde{Y}) \int_{0}^{\tilde{z}} d \tilde{z}^{\prime} M\left(\tilde{z}^{\prime}, b ; \tilde{Y}\right) .
\end{aligned}
$$

We are going to find solution inside the saturation region where function $M$ is small at large $\tilde{z}$. However, we need to re-write eq. (2.8) replacing it by

$$
\begin{aligned}
& \kappa \frac{\partial M(\tilde{z}, b ; \tilde{Y})}{\partial \tilde{z}}+\frac{\partial M(\tilde{z}, b ; \tilde{Y})}{\partial \tilde{Y}} \\
= & \left(\chi(\gamma)-\frac{1}{\gamma}\right) M(\tilde{z}, b ; \tilde{Y})-(\tilde{z}+\lambda) M(\tilde{z}, b ; \tilde{Y})+M(\tilde{z}, b ; \tilde{Y}) \int_{\tilde{z}}^{\infty} d \tilde{z}^{\prime} M\left(\tilde{z}^{\prime}, b ; \tilde{Y}\right)
\end{aligned}
$$

where

$$
\lambda=\int_{0}^{\infty} d \tilde{z}^{\prime} M\left(\tilde{z}^{\prime}, b ; \tilde{Y}\right)
$$

Neglecting the last term in this equation one can see that we need to solve the following linear equation

$$
\kappa \frac{\partial M(\bar{z}, b ; \tilde{Y})}{\partial \bar{z}}+\frac{\partial M(\bar{z}, b ; \tilde{Y})}{\partial \tilde{Y}}=\left(\chi(\gamma)-\frac{1}{\gamma}\right) M(\bar{z}, b ; \tilde{Y})-\bar{z} M(\bar{z}, b ; \tilde{Y})
$$

with $\gamma=\frac{\partial}{\partial \bar{z}}$ and $\bar{z}=\tilde{z}+\lambda$. Note, that the contribution of the last term is of the order of $(M(\tilde{z}, \tilde{Y}))^{2} \ll M(\tilde{z}, \tilde{Y})$ where $M$ is the solution to eq. (2.11), and can be neglected. On the other hand, $\lambda \sim \mathcal{O}(1)$ since the main contribution to this integral stems from $\tilde{z}^{\prime} \rightarrow 0$. Recall, $M(\tilde{z}=0)=\phi_{0}-\frac{1}{2}$. Hence, the term $\lambda M$ has to be taken into account. We will discuss corrections that stem from the last term in eq. (2.9) below in section 3.1.

First we find the geometrical scaling solution which depends only on $\bar{z}$. In this case eq. (2.11) takes the form

$$
\kappa \frac{d M(\bar{z}, b)}{d \bar{z}}=\left(\chi(\gamma)-\frac{1}{\gamma}\right) M(\bar{z}, b)-\bar{z} M(\bar{z}, b)
$$


The boundary condition for this equation we take

$$
N_{\tilde{z}}^{\prime}(\tilde{z}=0, b)=\frac{1}{2}+M(\tilde{z}=0, b)=\phi_{0}(b) \ll 1
$$

where $\phi_{0}(b)$ is the solution to the linear BFKL equation at $\tilde{z}=0 . \phi_{0}(b) \leq 1$ due to unitarity constraint and should be small to neglect that non-liner term at $\tilde{z}=0$.

Eq. (2.12) can be solved using the Mellin transform

$$
M(\bar{z}, b)=\int_{\epsilon-i \infty}^{\epsilon+i \infty} \frac{d \gamma}{2 \pi i} e^{\gamma \bar{z}} m(\gamma, b)
$$

where $m(\gamma, b)$ satisfies the equation:

$$
(\kappa \gamma-\chi(\gamma)+1 / \gamma) m(\gamma, b)=\frac{d m(\gamma, b)}{d \gamma} .
$$

The solution for $m(\gamma, b)$ takes the following form

$$
m(\gamma)=\exp \left(\int_{0}^{\gamma} d \gamma^{\prime}\left(\kappa \gamma^{\prime}-\chi\left(\gamma^{\prime}\right)+1 / \gamma^{\prime}\right)\right)
$$

and taking into account the explicit form of the BFKL kernel given by eq. (1.4) one can re-write eq. (2.16) in the form

$$
m(\gamma)=\exp \left(\kappa \gamma^{2} / 2-2 \psi(1) \gamma\right)\left(\frac{\gamma \Gamma(\gamma)}{\Gamma(1-\gamma)}\right)=\exp \left(\kappa \gamma^{2} / 2-2 \psi(1) \gamma\right) \frac{\Gamma(1+\gamma)}{\Gamma(1-\gamma)}
$$

Substituting eq. (2.17) into eq. (2.14) we obtain

$$
M(\bar{z}, b)=\int_{\epsilon-i \infty}^{\epsilon+i \infty} \frac{d \gamma}{2 \pi i} e^{\gamma \bar{z}+\kappa \gamma^{2} / 2} \frac{\Gamma(1+\gamma)}{\Gamma(1-\gamma)}
$$

where for $\bar{z}$ we use a new definition: $\bar{z}=\tilde{z}+\lambda-2 \psi(1)$.

One can see that in eq. (2.18) we cannot close the contour of integration in $\gamma$ neither on the left semi-plane nor on the right one. Introducing $\gamma=i \bar{\gamma}$ we reduce eq. (2.18) to the form

$$
M(\bar{z}, b)=\int_{-\infty}^{+\infty} \frac{d \bar{\gamma}}{2 \pi} e^{i \bar{\gamma} \bar{z}-\kappa \bar{\gamma}^{2} / 2} \frac{\Gamma(1+i \bar{\gamma})}{\Gamma(1-i \bar{\gamma})}
$$

For large $\tilde{z}$ and $\bar{\gamma}$ is large about $\tilde{z}$, we can use the approximation

$$
\Gamma(1+i \bar{\gamma}) \stackrel{\bar{\gamma} \gg 1}{\longrightarrow} \sqrt{2 \pi}|\bar{\gamma}|^{\frac{1}{2}} e^{-\frac{1}{2} \pi|\bar{\gamma}|}
$$

(see formula 8.328 of ref. [42]). Using eq. (2.20), eq. (2.19) takes the form

$$
M(\bar{z}, b)=\int_{-\infty}^{+\infty} \frac{d \bar{\gamma}}{2 \pi} e^{i \bar{\gamma} \bar{z}-\kappa \bar{\gamma}^{2} / 2}
$$

which is equal (see formulae 3.462(3), 9.246 of ref. [42])

$$
M(\bar{z}, b)=\frac{1}{\sqrt{2 \pi \kappa}} e^{-\frac{\bar{z}^{2}}{4 \kappa}} D_{0}\left(-\frac{\bar{z}}{\sqrt{\kappa}}\right)=\frac{1}{\sqrt{2 \pi \kappa}} e^{-\frac{\bar{z}^{2}}{2 \kappa}}
$$


where $D_{n}(z)=2^{-\frac{1}{2} n} e^{-\frac{z^{2}}{4}} H_{n}(z / \sqrt{2})$ is the parabolic cylinder function (see formulae 9.249.25 of ref. [42]).

Therefore we reproduce the solution of eq. (1.7). Choosing the coefficient in front of eq. (2.22) we can easily satisfy the boundary condition of eq. (2.13) which leads to the solution

$$
M(\bar{z}, b)=\left(\phi_{0}(b)-\frac{1}{2}\right) e^{-\frac{\bar{z}^{2}}{2 \kappa}}
$$

Actually, eq. (2.23) satisfies the condition $M(\bar{z}=0)=\phi_{0}(b)-\frac{1}{2}$ instead of $M(\tilde{z}=0)=$ $\phi_{0}(b)-\frac{1}{2}$. The correct matching we will make in section 3.3 .

\subsection{General solution and initial condition at $Y=Y_{A}$}

As has been mentioned we are not able to find the geometric scaling solution that satisfy both initial and boundary conditions given by eq. (1.8) and eq. (2.13). We need to solve a general eq. (2.11) to find such a solution. We start with re-writing initial condition of eq. (1.8) for function $M(\tilde{z}, b ; Y)$ in momentum representation. Actually, we replace eq. (1.8) by the simplified formula that looks as follows

$$
N\left(x^{2}, Y=Y_{A}\right)=1-\exp \left(-\frac{1}{4} x^{2} Q_{s}^{2}\left(Y=Y_{A}, b\right)\right)=1-\exp \left(-e^{\xi}\right)
$$

$\ln \left(\frac{1}{x \Lambda}\right)$ in eq. (1.8) plays a very important role for large values of momenta leading to the correct operator product expansion. However, inside the saturation region where all terms of the operator product expansion become of the same order, this log does not carry any significance. On the other hand the simplified formula of eq. (2.24) results in a dramatic simplification of the calculations which are nevertheless quite cumbersome and tedious. As one can see below that none of our qualitative results depends on the exact form of the boundary condition but the exact form of the dividing line in figure 2 .

Eq. (2.24) leads to the following initial condition

$$
\begin{aligned}
\frac{1}{2}+M\left(\tilde{z}, b ; \tilde{Y}=\tilde{Y}_{A}\right) & =\frac{d}{d \tilde{z}} \int \frac{d^{2} r}{r^{2}} e^{i \vec{k} \cdot \vec{r}}\left(1-\exp \left(-r^{2} Q_{s}^{2}\left(b ; \tilde{Y}=\tilde{Y}_{A}\right) / 4\right)\right) \\
& =\frac{d}{d \tilde{z}}\left(\frac{1}{2} \Gamma_{0}\left(\frac{k_{\perp}^{2}}{Q_{s}^{2}\left(b ; \tilde{Y}=\tilde{Y}_{A}\right)}\right)\right)=\frac{d}{d \tilde{z}}\left(\frac{1}{2} \Gamma_{0}\left(e^{-\tilde{z}}\right)\right) \\
& =\frac{1}{2} \exp \left(-e^{-\tilde{z}}\right) \\
M\left(\tilde{z}, b ; \tilde{Y}=\tilde{Y}_{A}\right) & =-\frac{1}{2}\left(1-\exp \left(-e^{-\tilde{z}}\right)\right)
\end{aligned}
$$

We solve eq. (2.11) using the double Mellin transform: viz.

$$
M(\bar{z}, b ; \tilde{Y})=\int_{\epsilon-i \infty}^{\epsilon+i \infty} \frac{d \omega}{2 \pi i} \int_{\epsilon-i \infty}^{\epsilon+i \infty} \frac{d \gamma}{2 \pi i} e^{\omega\left(\tilde{Y}-\tilde{Y}_{A}\right)+\gamma \bar{z}} m(\omega, \gamma ; b)
$$


For $m(\omega, \gamma ; b)$ the equation takes the form ${ }^{2}$

$$
\left(\omega+\kappa \gamma-\chi(\gamma)+\frac{1}{\gamma}\right) m(\omega, \gamma)=\frac{\partial m(\omega, \gamma)}{\partial \gamma}
$$

Solution to eq. (2.27) can be written in the form

$$
M(\bar{z}, \tilde{Y})=\int_{\epsilon-i \infty}^{\epsilon+i \infty} \frac{d \omega}{2 \pi i} e^{\omega\left(\tilde{Y}-\tilde{Y}_{A}\right)} \int_{\epsilon-i \infty}^{\epsilon+i \infty} \frac{d \gamma}{2 \pi i} I(\omega) \exp \left(\omega \gamma+\gamma \bar{z}+\kappa \gamma^{2} / 2\right) \frac{\Gamma(1+\gamma)}{\Gamma(1-\gamma)}
$$

where function $I(\omega)$ has to be found from eq. (2.25). At $Y=Y_{A}$ eq. (2.25) can be written as

$$
\begin{aligned}
& M\left(\bar{z}, \tilde{Y}=\tilde{Y}_{A}\right) \\
= & \int_{\epsilon-i \infty}^{\epsilon+i \infty} \frac{d \omega}{2 \pi i} \int_{\epsilon-i \infty}^{\epsilon+i \infty} \frac{d \gamma}{2 \pi i} I(\omega) \exp \left(\omega \gamma+\gamma \bar{z}+\kappa \gamma^{2} / 2\right) \frac{\Gamma(1+\gamma)}{\Gamma(1-\gamma)} \\
= & \int_{\epsilon-i \infty}^{\epsilon+i \infty} \frac{d \gamma}{2 \pi i} J(\gamma) \exp \left(\gamma \tilde{z}+\kappa \gamma^{2} / 2-2 \psi(1) \gamma+\lambda \gamma\right) \frac{\Gamma(1+\gamma)}{\Gamma(1-\gamma)}
\end{aligned}
$$

One can see that

$$
J(\gamma)=\int_{\epsilon-i \infty}^{\epsilon+i \infty} \frac{d \omega}{2 \pi i} e^{\omega \gamma} I(\omega)=\frac{1}{2} \frac{\Gamma(1-\gamma)}{\Gamma(1+\gamma)}\left(\Gamma(\gamma)-\frac{1}{\gamma}\right) \exp \left(-\frac{\kappa \gamma^{2}}{2}+2 \psi(1) \gamma-\lambda \gamma\right)
$$

leads to eq. (2.29). Indeed, substituting eq. (2.30) into eq. (2.29) one obtains eq. (2.25) closing contour in $\gamma$ over negative $\gamma$-s. For $\tilde{Y}>\tilde{Y}_{A}$ the solution takes the form ${ }^{3}$

$$
\begin{gathered}
M(\bar{z}, \tilde{Y})=\int_{\epsilon-i \infty}^{\epsilon+i \infty} \frac{d \gamma}{2 \pi i} J(Y+\gamma) \exp \left(\gamma \bar{z}+\kappa \gamma^{2} / 2\right) \frac{\Gamma(1+\gamma)}{\Gamma(1-\gamma)} \\
=\frac{1}{2} \int_{\epsilon-i \infty}^{\epsilon+i \infty} \frac{d \gamma}{2 \pi i} \exp \left(\gamma \tilde{z}-\gamma \kappa \tilde{Y}-\frac{1}{2} \kappa \tilde{Y}^{2}+(2 \psi(1)-\lambda) \tilde{Y}\right) \frac{\Gamma(1+\gamma)}{\Gamma(1-\gamma)} \\
\quad \times \frac{\Gamma(1-\gamma-\tilde{Y})}{\Gamma(1+\gamma+\tilde{Y})}\left(\Gamma(\gamma+\tilde{Y})-\frac{1}{\gamma+\tilde{Y}}\right) \\
=\frac{1}{2} e^{-\frac{1}{2} \kappa \tilde{Y}^{2}} \int_{\epsilon-i \infty}^{\epsilon+i \infty} \frac{d \gamma}{2 \pi i} \exp (-\gamma \tilde{\xi}+(2 \psi(1)-\lambda) \tilde{Y}) \frac{\Gamma(1+\gamma)}{\Gamma(1-\gamma)} \\
\times \frac{\Gamma(1-\gamma-\tilde{Y})}{\Gamma(1+\gamma+\tilde{Y})}\left(\Gamma(\gamma+\tilde{Y})-\frac{1}{\gamma+\tilde{Y}}\right) \\
=-\frac{1}{2} e^{-\frac{1}{2} \kappa \tilde{Y}^{2}+(2 \psi(1)-\lambda) \tilde{Y}} \int_{\epsilon-i \infty}^{\epsilon+i \infty} \frac{d \gamma}{2 \pi i} \exp (-\gamma \tilde{\xi}) \frac{\Gamma(1+\gamma)}{\Gamma(1-\gamma)} \\
\times \frac{\Gamma(-\gamma-\tilde{Y})}{\Gamma(\gamma+\tilde{Y})}\left(\Gamma(\gamma+\tilde{Y})-\frac{1}{\gamma+\tilde{Y}}\right) \\
=-\frac{1}{2} e^{-\frac{1}{2} \kappa \tilde{Y}^{2}+(2 \psi(1)-\lambda) \tilde{Y}}\left\{I_{1}(\tilde{Y}, \tilde{\xi})-I_{2}(\tilde{Y}, \tilde{\xi})\right\}
\end{gathered}
$$

\footnotetext{
${ }^{2}$ We omitted argument $b$ in $m(\omega, \gamma ; b)$ since our equations do not depend on $b$ and it enters only through the initial and boundary conditions.

${ }^{3}$ For simplicity we use $\tilde{Y}=\tilde{Y}-\tilde{Y}_{A}$ to the end of this section. We hope that using the same letter $Y$ for both variables, will not cause any inconvenience.
} 
Since $\tilde{\xi}<0$ we can take the integrals over $\gamma$ in $I_{1}(\tilde{Y}, \tilde{\xi})$ and in $I_{2}(\tilde{Y}, \tilde{\xi})$ closing contours of integrations on the left semi-plane. In $I_{1}(\tilde{\xi})$ and $I_{2}(\tilde{\xi})$ we have two sets of poles: $\gamma=-n-1$ from $\Gamma(1+\gamma)$ and $\gamma=n-[\tilde{Y}]$ where $[\tilde{Y}]$ is the integer part or floor function of $\tilde{Y}$, from $\Gamma(-\gamma-\tilde{Y})$. These sets lead to the following contributions to $I_{1}(\tilde{Y}, \tilde{\xi})$ and to $I_{2}(\tilde{Y}, \tilde{\xi}):$

$$
\begin{aligned}
I_{1}(\tilde{Y}, \tilde{\xi}) & =I_{1}^{1}(\tilde{Y}, \tilde{\xi})+I_{1}^{2}(\tilde{Y}, \tilde{\xi}) \\
I_{1}^{1}(\tilde{Y}, \tilde{\xi}) & =\sum_{n=0}^{\infty} \frac{(-1)^{n}}{n !} \frac{\Gamma(n+1-\tilde{Y})}{\Gamma(n+2)} e^{(n+1) \tilde{\xi}} \\
& =e^{\tilde{\xi}} \Gamma(1-\tilde{Y}){ }_{1} F_{1}\left(1-\tilde{Y}, 2,-e^{\tilde{\xi}}\right) ; \\
I_{1}^{2}(\tilde{Y}, \tilde{\xi}) & =e^{\tilde{\xi} \tilde{Y}} \sum_{n=0}^{[\tilde{Y}]} \frac{(-1)^{n}}{n !} \frac{\Gamma(n+1-\tilde{Y})}{\Gamma(1-n+\tilde{Y})} e^{-n \tilde{\xi}} ; \\
I_{2}(\tilde{Y}, \tilde{\xi}) & =I_{2}^{1}(\tilde{Y}, \tilde{\xi})+I_{2}^{2}(\tilde{Y}, \tilde{\xi}) ; \\
I_{2}^{1}(\tilde{Y}, \tilde{\xi}) & =\sum_{n=0}^{\infty} \frac{(-1)^{n}}{n !} \frac{\Gamma(n+1-\tilde{Y})}{\Gamma(n+2) \Gamma(\tilde{Y}-n)} e^{(n+1) \tilde{\xi}} \\
& =e^{\tilde{\xi}} \frac{\Gamma(1-\tilde{Y})}{\Gamma(\tilde{Y})}{ }_{2} F_{1}\left(1-\tilde{Y},-\tilde{Y}, 2,-e^{\tilde{\xi}}\right) \\
I_{2}^{2}(\tilde{Y}, \tilde{\xi}) & =e^{\tilde{\xi} \tilde{Y}} \sum_{n=0}^{[\tilde{Y}]} \frac{(-1)^{n}}{n !} \frac{\Gamma(n+1-\tilde{Y})}{\Gamma(1-n+\tilde{Y}) \Gamma(n+1)} e^{-n \tilde{\xi}} ;
\end{aligned}
$$

In eqs. (2.32)-(2.35) ${ }_{1} F_{1}(\alpha, \beta, t)$ is the confluent hypergeometric function (another notation is $\Phi(\alpha, \beta, t)$, see formulae 9.2 of ref. [42]) and ${ }_{2} F_{1}(\alpha, \beta, \gamma, t)$ is the hypergeometric function (see formulae 9.1 of ref. [42]).

For matching of this solution with the solution given by eq. (2.23) we need to know the asymptotic behaviour of eqs. $(2.32)-(2.35)$ at large values of $\tilde{Y}$. Using Kummer's transformation: ${ }_{1} F_{1}(\alpha, \beta, t)=e^{t}{ }_{1} F_{1}(\beta-\alpha, \beta,-t)$ we can re-write eq. (2.32) in the form

$$
\begin{aligned}
& I_{1}^{1}(\tilde{Y}, \tilde{\xi})=e^{\tilde{\xi}} \Gamma(1-\tilde{Y}) \exp \left(-e^{\tilde{\xi}}\right){ }_{1} F_{1}\left(1+\tilde{Y}, 2, e^{\tilde{\xi}}\right) \\
& \stackrel{\tilde{Y} \gg 1}{\longrightarrow} e^{\tilde{\xi}} \exp \left(-e^{\tilde{\xi}}\right) \Gamma(1-\tilde{Y}) \sum_{n=0}^{\infty} \frac{\tilde{Y}^{n}}{n !(n+1) !} e^{n \tilde{\xi}} \\
& \quad=e^{\tilde{\xi}} \exp \left(-e^{\tilde{\xi}}\right) \frac{\Gamma(1-\tilde{Y})}{2 \sqrt{\tilde{Y} e^{\tilde{\xi}}}} I_{1}\left(2 \sqrt{\tilde{Y} e^{\tilde{\xi}}}\right)
\end{aligned}
$$

where $I_{1}(t)$ is the modified Bessel function of the first kind (see formulae 8.445-8.451 of 
ref. [42]). Using their asymptotic behaviour at large values of the argument we obtain

$$
I_{1}^{1}(\tilde{Y}, \tilde{\xi}) \stackrel{\tilde{Y} \gg 1}{\longrightarrow} e^{\tilde{\xi}} \exp \left(-e^{\tilde{\xi}}\right) e^{-\tilde{Y}(\ln \tilde{Y}-1)} \frac{1}{\sqrt{2 \pi(4 \tilde{Y} \exp (\tilde{\xi}))^{3}}} \exp (2 \sqrt{\tilde{Y} \exp (\tilde{\xi})})
$$

We replace eq. (2.33) by the integral, i.e.

$$
I_{1}^{2}(\tilde{Y}, \tilde{\xi}) \rightarrow e^{\tilde{\xi} \tilde{Y}} \int_{0}^{\tilde{Y}} d t \frac{\Gamma(1+t-\tilde{Y})}{\Gamma(1-t+\tilde{Y}) \Gamma(1+t)} e^{-\tilde{\xi} t+i \pi t}
$$

The steepest decent method leads to the following contribution

$$
I_{1}^{2}(\tilde{Y}, \tilde{\xi}) \rightarrow \sqrt{\pi\left(\tilde{Y} e^{\tilde{\xi}}\right)^{\frac{1}{2}}} e^{-\tilde{Y} \ln \tilde{Y}}
$$

at the saddle point $t_{S P}$ which can be found from the equation

$$
-\ln t_{S P}+2 \ln \left(\tilde{Y}-t_{S P}-1\right)-\tilde{\xi}=0 ; t_{S P}=\tilde{Y}-\sqrt{\tilde{Y}} e^{\frac{1}{2}} \tilde{\xi} \text { at } \tilde{Y} \gg 1
$$

The large $\tilde{Y}$ behaviour of eq. (2.34) can be obtained using the transformation ${ }_{2} F_{1}(\alpha, \beta, \gamma, t)$ $=(1-t)^{\gamma-\alpha-\beta}{ }_{2} F_{1}(\gamma-\alpha, \gamma-\beta, \gamma, t)$ (see formulae 9.131 of ref. [42])

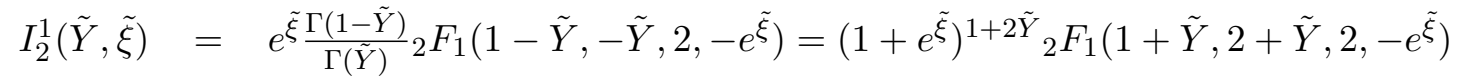

$$
\begin{aligned}
& \stackrel{\tilde{Y} \gg 1}{\longrightarrow} \sum_{n=0}^{\infty} \frac{(\Gamma(\tilde{Y}+n))^{2}}{(\Gamma(\tilde{Y}))^{2}} \frac{\left(-e^{\tilde{\xi}}\right)^{n}}{n !(n+1) !}=e^{\tilde{\xi}} e^{-2 \tilde{Y}(\ln \tilde{Y}-1)} \frac{1}{2 \tilde{Y} e^{\frac{1}{2} \tilde{\xi}}} J_{1}\left(2 \tilde{Y} e^{\frac{1}{2} \tilde{\xi}}\right)
\end{aligned}
$$

and knowing the asymptotic representation of Bessel function we get

$$
\begin{array}{r}
I_{2}^{1}(\tilde{Y}, \tilde{\xi}) \stackrel{\tilde{Y} \gg 1}{\longrightarrow} \frac{1}{2} e^{\tilde{\xi}} e^{-2 \tilde{Y}(\ln \tilde{Y}-1)} \frac{1}{\left(\tilde{Y} e^{\left.\frac{1}{2} \tilde{\xi}\right)^{\frac{3}{2}}}\right.}\left\{\cos \left(\left\{8 \tilde{Y} e^{\frac{\tilde{\xi}}{2}}-3 \pi\right\} / 4\right)\left[\frac{\Gamma(7 / 2)}{\Gamma(-1 / 2)}+O\left(\left(\tilde{Y}^{2} e^{\tilde{\xi}}\right)^{-1}\right)\right]\right. \\
\left.-\sin \left(\left\{8 \tilde{Y} e^{\frac{\tilde{\xi}}{2}}-3 \pi\right\} / 4\right)\left[\frac{\Gamma(9 / 2)}{\Gamma(-3 / 2)}+O\left(\left(\tilde{Y}^{2} e^{\tilde{\xi}}\right)^{-1}\right)\right]\right\}
\end{array}
$$

Finally, we replace eq. (2.35) by the integral to estimate the large $Y$ dependence of this term, i.e.

$$
I_{2}^{2}(\tilde{Y}, \tilde{\xi}) \rightarrow e^{\tilde{\xi} \tilde{Y}} \int_{0}^{\tilde{Y}} d t \frac{\Gamma(1+t-\tilde{Y})}{\Gamma(1-t+\tilde{Y}) \Gamma^{2}(1+t)} e^{-\tilde{\xi} t+i \pi t}
$$

Taking the integral by the steepest decent method in the same way as in eq. (2.41) we obtain the equation for the saddle point $t_{S P}$ :

$$
-2 \ln t_{S P}+2 \ln \left(\tilde{Y}-t_{S P}-1\right)-\tilde{\xi}=0 ; t_{S P}=\frac{\tilde{Y}-1}{1+e^{\frac{1}{2} \tilde{\xi}}}
$$




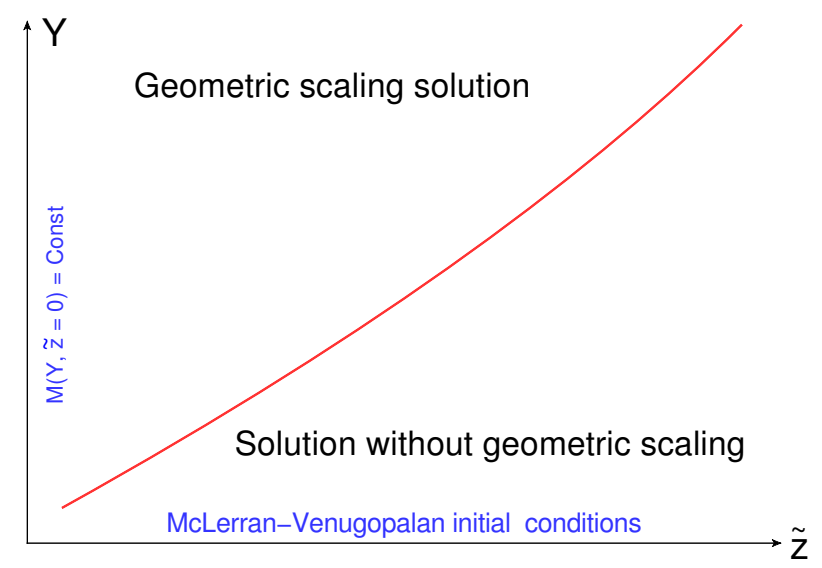

Figure 2. Saturation region: two domains in which there is the geometric scaling behaviour of the solution and there is no such behaviour. The border red line is eq. (2.45).

and

$$
I_{2}^{2}(\tilde{Y}, \tilde{\xi}) \rightarrow \sqrt{\frac{\pi(\tilde{Y}-1) e^{\frac{1}{2} \tilde{\xi}}}{4\left(1-e^{\tilde{\xi}}\right)}} e^{-2 \tilde{Y} \ln \tilde{Y}+\tilde{Y} \tilde{\xi} \frac{e^{\frac{1}{2} \tilde{\xi}}}{1+e^{\frac{1}{2} \tilde{\xi}}}}
$$

Comparing eq. (2.37), eq. (2.39), eq. (2.41) and eq. (2.44) we see that at large values of $\tilde{Y}$ solutions of eq. (2.23) and of eq. (2.31) match each other on the line $\tilde{\xi}=-\ln \tilde{Y}$ which can be translate as the line in $(Y, \tilde{z})$ plane (see figure 2 ):

$$
\tilde{z}=\bar{\alpha}_{S} \kappa Y+\ln \tilde{Y}
$$

\section{Matching two solutions: at small $\tilde{z}$ and at large $\tilde{z}$}

\subsection{Corrections at large $\tilde{z}$}

In this section we are going to find the first correction to the non-linear equation (see eq. (2.9)) deeply in the saturation region where we expect that solution has a geometric scaling behaviour, or in other words, it is a function of $\tilde{z}$. One can see that the equation for first correction takes the following form after substituting $M=M^{(0)}+M^{(1)}$ into eq. (2.9):

$$
\kappa \frac{d M^{(1)}(\bar{z})}{d \bar{z}}=\left\{\chi(\gamma)-\frac{1}{\gamma}\right\} M^{(1)}(\bar{z})-\bar{z} M^{(1)}(\bar{z})+M^{(0)}(\bar{z}) \int_{\bar{z}}^{\infty} d \bar{z}^{\prime} M^{(0)}\left(\bar{z}^{\prime}\right)
$$

where $\lambda=\int_{0}^{\infty} M^{(0)}\left(\tilde{z}^{\prime}\right) d \tilde{z}^{\prime}=A \sqrt{\frac{\pi \kappa}{2}}$.

In eq. (3.1) $M^{(0)}$ is the solutions to eq. (2.12) that takes the form

$$
M^{(0)}(\bar{z})=A \exp \left(-\bar{z}^{2} /(2 \kappa)\right)
$$

which we will use below. It should be noted that $A<0$ due to unitarity constraints; and eq. (2.23) for $A$ was derived from the matching of this solution at $\tilde{z}=0$. Here, we wish to suggest a better procedure for matching. 
After taking the integral the last term of eq. (3.1) can be reduced to the form

$$
M^{(0)}(\bar{z}) \int_{\bar{z}}^{\infty} d \bar{z}^{\prime} M^{(0)}\left(\bar{z}^{\prime}\right)=M^{(0)}(\bar{z}) A \sqrt{\frac{\pi \kappa}{2}} \operatorname{Erfc}\left(\frac{\bar{z}}{\sqrt{2 \kappa}}\right) \stackrel{z \gg 1}{\longrightarrow} A^{2} \frac{\kappa}{\bar{z}} e^{-\frac{\bar{z}^{2}}{\kappa}}
$$

Using the Mellin transform of eq. (2.14) we obtain eq. (3.1) in the form:

$$
\left(\kappa \gamma-\chi(\gamma)+\frac{1}{\gamma}+\lambda\right) m^{(1)}(\gamma)=\frac{d m^{(1)}(\gamma)}{d \gamma}+2 A^{2} \frac{\sqrt{\pi \kappa}}{\gamma} e^{\kappa \gamma^{2} / 4}
$$

The solution to eq. (3.4) takes the form (see ref. [43, 44])

$$
\begin{aligned}
m^{(1)}(\gamma) & =m^{(0)}(\gamma) A^{2} \int_{\gamma}^{\infty} \frac{d \gamma^{\prime}}{m^{(0)}\left(\gamma^{\prime}\right)} \frac{\sqrt{\pi \kappa}}{\gamma^{\prime}} e^{\kappa \gamma^{\prime 2} / 4} \\
& =m^{(0)}(\gamma) A^{2} \sqrt{\pi \kappa} \int_{\gamma}^{\infty} \frac{d \gamma^{\prime}}{\gamma^{\prime}} \frac{\Gamma\left(1-\gamma^{\prime}\right)}{\Gamma\left(1+\gamma^{\prime}\right)} e^{-\kappa \gamma^{\prime 2} / 4}
\end{aligned}
$$

where $m^{(0)}$ is given by eq. (2.17). As has been discussed in the integral of eq. (2.14) we expect that large $\gamma^{\prime}$ 's will be essential. In eq. (3.5) the typical $d \gamma^{\prime} \sim 1 / \sqrt{\kappa} \ll \gamma$ and we can replace this integral by

$$
\begin{aligned}
m^{(1)}(\gamma) & =2 m^{(0)}(\gamma) A^{2} \sqrt{\pi \kappa} \frac{\Gamma(1-\gamma)}{\Gamma(1+\gamma)} \int_{\gamma}^{\infty} \frac{d \gamma^{\prime}}{\gamma^{\prime}} e^{-\kappa \gamma^{\prime 2} / 4} \\
& =-2 m^{(0)}(\gamma) A^{2} \sqrt{\pi \kappa} \frac{\Gamma(1-\gamma)}{\Gamma(1+\gamma)} E i\left(-\kappa \gamma^{2} / 4\right)
\end{aligned}
$$

See ref. [42]: formula 3.352(2) for the last integration and formula 8.21 for the exponential integral $E(x)$. For large $\gamma$ eq. (3.6) takes the form

$$
m^{(1)}(\gamma)=m^{(0)}(\gamma) A^{2} \sqrt{\pi \kappa} \frac{\Gamma(1-\gamma)}{\Gamma(1+\gamma)} \frac{4}{\kappa \gamma^{2}} \exp \left(-\kappa \gamma^{2} / 4\right)
$$

Plugging eq. (3.7) in eq. (2.14) we obtain $M^{(1)}(\bar{z})$ in the form

$$
\begin{aligned}
M^{(1)}(\bar{z}) & =A^{2} \frac{4 \sqrt{\pi \kappa}}{\kappa} \int_{\epsilon-i \infty}^{\epsilon+i \infty} \frac{d \gamma}{2 \pi i} \frac{1}{\gamma^{2}} e^{\gamma \bar{z}+\frac{\kappa \gamma^{2}}{4}} \\
& =-A^{2} e^{-\bar{z}^{2} / 2 \kappa} D_{-2}\left(\bar{z} \sqrt{\frac{2}{\kappa}}\right) \stackrel{\bar{z} \gg 1}{\longrightarrow}-A^{2} \frac{\kappa}{\bar{z}^{2}} e^{-\bar{z}^{2} / \kappa}
\end{aligned}
$$

where $D_{p}(z)$ is the parabolic cylinder function (see formulae 9.24-9.25 of ref. [20]).

\subsection{Corrections at small $\tilde{z}$}

In the region of small $\tilde{z}$ we can solve eq. (2.2) for the amplitude $N(\tilde{z})$ noting that the geometric scaling solution to the BFKL equation occurs at $\gamma=\gamma_{c r}$. In the vicinity of the saturation scale the linear BFKL equation can be simplified and replaced by

$$
\frac{d \widetilde{N}(\tilde{z})}{d \tilde{z}}=\left(1-\gamma_{c r}\right) \tilde{N}(\tilde{z})
$$


One can see that this equation leads to $\widetilde{N}(\tilde{z}) \propto \exp \left(\left(1-\gamma_{c r}\right) \tilde{z}\right)$. The solution to the BFKL equation with full kernel in the vicinity of the saturation scale takes the form [24-27]

$$
\tilde{N}\left(\tilde{z} ; Q_{s}(Y)\right)=\phi_{0} e^{\left(1-\gamma_{c r}\right) \tilde{z}} \exp \left(-\frac{\tilde{z}^{2}}{2 \frac{\chi_{\gamma \gamma}^{\prime \prime}\left(\gamma_{c r}\right)}{\kappa} \ln \left(Q_{s}^{2}(Y) / Q_{s}^{2}(0)\right)}\right)
$$

Therefore, we can trust eq. (3.9) only for

$$
\tilde{z} \ll \sqrt{2 \frac{\chi_{\gamma \gamma}^{\prime \prime}\left(\gamma_{c r}\right)}{\kappa} \ln \left(Q_{s}^{2}(Y) / Q_{s}^{2}(0)\right)}=9.9 \sqrt{Y}
$$

For such $\tilde{z}$ the non-linear equation (see eq. (2.2)) can be re-written in the form

$$
\kappa \frac{d \widetilde{N}(\tilde{z})}{d \tilde{z}}=\kappa\left(1-\gamma_{c r}\right) \widetilde{N}(\tilde{z})-\widetilde{N}^{2}(\tilde{z})
$$

The solution to this equation that satisfies the initial condition $\tilde{N}(\tilde{z}=0)=N_{0}$ takes the following form

$$
\widetilde{N}(\tilde{z})=\frac{\kappa\left(1-\gamma_{c r}\right) N_{0}}{N_{0}-\left(N_{0}-\kappa\left(1-\gamma_{c r}\right)\right) e^{-\left(1-\gamma_{c r}\right) \tilde{z}}}
$$

As we will discuss in the next subsection the scattering amplitude in the coordinate space is equal to

$$
N(z)=\left.2 \frac{d \tilde{N}(\tilde{z})}{d \tilde{z}}\right|_{\tilde{z}=z}=-2 \frac{\kappa\left(1-\gamma_{c r}\right)^{2}\left(N_{0}-\kappa\left(1-\gamma_{c r}\right)\right) N_{0} e^{-\left(1-\gamma_{c r}\right) z}}{\left(N_{0}-\left(N_{0}-\kappa\left(1-\gamma_{c r}\right)\right) e^{-\left(1-\gamma_{c r}\right) z}\right)^{2}}
$$

We will use this equation in the matching procedure described below in the next section.

\subsection{Matching procedure}

In this subsection we would like to discuss matching of two solutions at small and large $\tilde{z}$. First, we calculate the scattering amplitude of two dipoles in the coordinate representation where we know that at large $z$ this amplitude $N(z) \rightarrow 1$.

From eq. (2.1) we see that

$$
2 \pi \widetilde{N}\left(k_{\perp}, b ; Y\right)=2 \pi \int x d x \frac{J_{0}\left(k_{\perp} x\right)}{x^{2}} N(x, b ; Y)
$$

The main contribution to the integral over $x$ stems from $k_{\perp} x \leq 2.4$, where 2.4 is the zero of $J_{0}\left(k_{\perp} x\right)\left(J_{0}\left(k_{\perp} x=2.4\right)=0\right)$, and therefore, we can replace the integral by

$$
\widetilde{N}\left(k_{\perp}, b ; Y\right)=\frac{1}{2} \int_{0}^{\frac{2.4^{2}}{k_{\perp}^{2}}} \frac{d x^{2}}{x^{2}} N(x, b ; Y)=\frac{1}{2} \int_{-\infty}^{\ln \left(2.4^{2} / k_{\perp}^{2}\right)} d \ln x^{2} N\left(x^{2}, b, Y\right)
$$

or

$$
N(x, b, Y)=\left.2 \frac{d \widetilde{N}\left(k_{\perp}, b ; Y\right)}{d \ln \left(2.4^{2} / k_{\perp}^{2}\right)}\right|_{x=2.4 / k_{\perp}}
$$




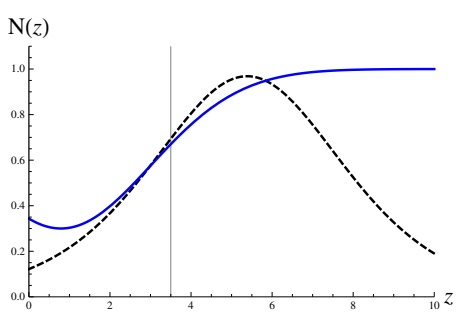

figure 3 -a

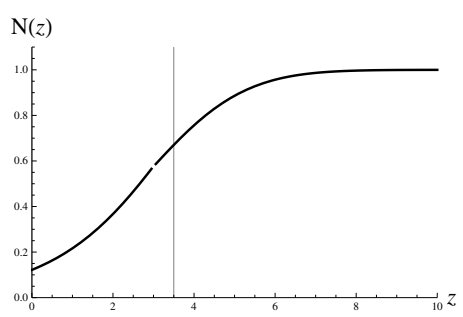

figure $3-\mathrm{d}$

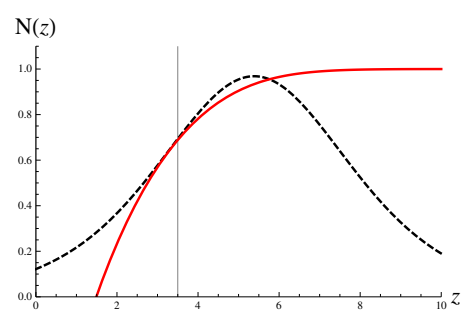

figure $3-b$

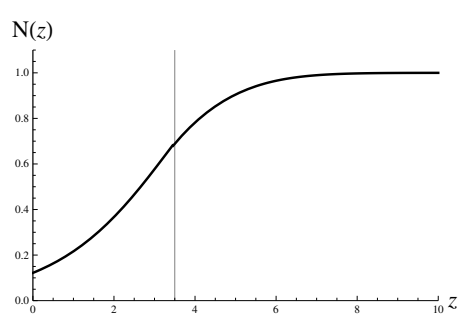

figure 3-e

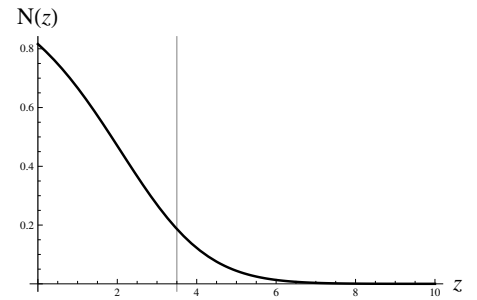

figure $3-\mathrm{c}$

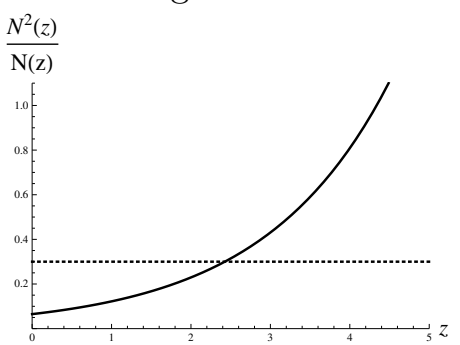

figure $3-\mathrm{f}$

Figure 3. The scattering amplitude $N(z)$ in the coordinate representation: (figure 3-a) matching of two solutions: $N(z)$ at $z<1$ ( dashed black b line) and $N^{(0)}(z) z \gg 1$ ( solid blue line) and (figure 3-b) matching of two solutions: $N(z)$ at $z<1$ ( dashed black line) and $N^{(1)}(z) z \gg 1$ ( solid red line). The ratio $M^{(1)}(z) / M^{(0)}(z)$ is shown in figure 3-c. The value of $A$ is chosen to be equal to 0.69 for figure 3 -a and $A=0.65$ for figure 3-b. The vertical line marks $z_{m}=3.5$, for $z>z_{m}$ the corrections due to $M^{(1)}$ are small (less 30\%), while for $z<z_{m}$ the non-linear contributions to the amplitude is less than $30 \%$ (see figure 3 -f where the ratio of the contribution proportional to $N^{2}(z)$ to the contribution proportional to $N(z)$ is shown). Figure 3-d and figure 3-e show the resulting amplitude due to the matching procedure presented in figure 3 -a and figure 3 -b . The value of $N_{0}$ is chosen to be equal 0.1 in the picture and $z=\ln \left(x^{2} Q_{s}^{2} /(2.4)^{2}\right)$.

For the solution with the geometric scaling behaviour eq. (3.17) takes the form

$$
N(z)=\left.2 \frac{d \tilde{N}(\tilde{z})}{d \tilde{z}}\right|_{z=\tilde{z}}
$$

Note, that in eq. (3.18) we redefined $z$ as $z=\ln \left(x^{2} Q_{s}^{2} /(2.4)^{2}\right)$ including factor $2.4^{2}$ in comparison with eq. (1.6). It should be noted that in McLerran-Venugopalan formula (eqs. (1.8) and (2.24)) $z$ is defined as $z=\ln \left(x^{2} Q_{s}^{2} / 4\right)$.

Bearing eq. (3.18) in mind we can formulate the matching procedure in the following way

$$
\begin{aligned}
& N_{z<1}\left(z_{m}, N_{0}\right)=N_{z \gg 1}^{(0)}\left(z_{m}, A\right)=1+2 M^{(0)}\left(z_{m}, A\right) \\
& \frac{d N_{z<1}\left(z_{m}, A\right)}{d z}=\frac{d N_{z \gg 1}^{(0)}\left(z_{m}, A\right)}{d z}=+2 \frac{d M^{(0)}\left(z_{m}, A\right)}{d z}
\end{aligned}
$$

where we denote $N_{z<1}$ the solution for small $z$ while $N_{z \gg 1}$ is the solution at large $z$.

One can see from figure 3 -a that we cannot find the solution to both eq. (3.19), but the second equation in eq. (3.19) is almost satisfied. Actually, this matching supports the approach developed in ref. [27]. From figure 3-b one can see that the next to leading 
corrections at large $z$ drastically change the situation leading to the fact that both equations of eq. (3.20) are satisfied.

$$
\begin{aligned}
N_{z<1}\left(z_{m}, N_{0}\right) & =N_{z \gg 1}^{(1)}\left(z_{m}, A\right)=1+2 M^{(0)}\left(z_{m}, A\right)+2 M^{(1)}\left(z_{m}, A\right) \\
\frac{d N_{z<1}\left(z_{m}, N_{0}\right)}{d z} & =\frac{d N_{z \gg 1}^{(1)}\left(z_{m}, A\right)}{d z}=2 \frac{d\left(M^{(0)}\left(z_{m}, A\right)+M^{(1)}\left(z_{m}, A\right)\right)}{d z}
\end{aligned}
$$

It is interesting to note that the solution to eq. (3.20): $z_{m}=3.5$ and $A=0.65$, gives such values of these parameters that matching occurs in the region where the next-to-leading corrections to asymptotical contribution $\left(M^{(1)}\right)$ is less or about of $30 \%$ $\left(M^{(1)}\left(z_{m}\right) / M^{(0)}\left(z_{m}\right) \leq 0.3\right)$. On the other hand the non-linear corrections are not large $(<30 \%)$ and we can trust the simplified eq. (3.12), since $z_{m}$ satisfies eq. (3.10) even at $Y=1$.

In general we consider this matching as a strong argument for the procedure suggested in ref. [27] for finding the approximate solution of the non-linear equation which is suited for phenomenology.

\section{Conclusions}

In this paper we show that at large $z$ the solution to Balitsky-Kovchegov equation takes the following form

$$
N(z)=1-A \exp \left(-\frac{(z+A \sqrt{\kappa \pi / 8}-2 \psi(1))^{2}}{2 \kappa}\right)
$$

which is the same as solution given in ref. [23] at $z \gg 1$. However, the asymptotic behaviour of the solution depends on different variable $z+A \sqrt{\kappa \pi / 8}-2 \psi(1)$ while the solution at small $z$ in the vicinity of the saturation scale is determined by $z$. This observation, we believe, is essential for understanding the matching of the solutions at small and large $z$ and for searching the solution for intermediate $z$. We would like to note again that $z=\ln \left(x^{2} Q_{s}^{2} /(2.4)^{2}\right)$.

We found the solution in the entire kinematic region at large $z$ which satisfies the McLerran-Venugopalan initial condition in the simplest form of eq. (2.24). This problem has been discussed in refs. [35, 36] in the case of simplified kernels but here we give the solution for the full BFKL kernel. The fact that we use eq. (2.24) instead of eq. (1.8) does not change our conclusion that the saturation region has to be divided in two: the first with the geometric scaling behaviour for large values of $Y$ and the second where we do not expect the geometric scaling behaviour. The dividing line depends on the boundary conditions and it is given in the paper for eq. (2.24). It should be mentioned that eq. (2.24) is widely used in the phenomenological applications [22].

The next-to-leading in the region of large $z$ has been calculated and it is demonstrated that this correction change crucially the matching with the solution in the vicinity of the saturation scale.

We hope that this paper will be useful for finding general features of the behaviour of the dipole scattering amplitude in the saturation region. 


\section{Acknowledgments}

We thank our colleagues at UTFSM and Tel Aviv university for encouraging discussions. This research was supported by the BSF grant 2012124 and by the Fondecyt (Chile) grants 1140842 and 1120920 and DGIP USM grant 11.13.12 .

Open Access. This article is distributed under the terms of the Creative Commons Attribution License (CC-BY 4.0), which permits any use, distribution and reproduction in any medium, provided the original author(s) and source are credited.

\section{References}

[1] L.V. Gribov, E.M. Levin and M.G. Ryskin, Semihard Processes in QCD, Phys. Rept. 100 (1983) 1 [INSPIRE].

[2] A.H. Mueller and J.-w. Qiu, Gluon Recombination and Shadowing at Small Values of $x$, Nucl. Phys. B 268 (1986) 427 [inSPIRE].

[3] L.D. McLerran and R. Venugopalan, Computing quark and gluon distribution functions for very large nuclei, Phys. Rev. D 49 (1994) 2233 [hep-ph/9309289] [INSPIRE].

[4] L.D. McLerran and R. Venugopalan, Gluon distribution functions for very large nuclei at small transverse momentum, Phys. Rev. D 49 (1994) 3352 [hep-ph/9311205] [INSPIRE].

[5] L.D. McLerran and R. Venugopalan, Green's functions in the color field of a large nucleus, Phys. Rev. D 50 (1994) 2225 [hep-ph/9402335] [INSPIRE].

[6] A. Ayala, J. Jalilian-Marian, L.D. McLerran and R. Venugopalan, Quantum corrections to the Weizsacker-Williams gluon distribution function at small x, Phys. Rev. D 53 (1996) 458 [hep-ph/9508302] [INSPIRE].

[7] L.D. McLerran and R. Venugopalan, Fock space distributions, structure functions, higher twists and small x, Phys. Rev. D 59 (1999) 094002 [hep-ph/9809427] [INSPIRE].

[8] A.H. Mueller, Soft gluons in the infinite momentum wave function and the BFKL Pomeron, Nucl. Phys. B 415 (1994) 373 [inSPIRE].

[9] A.H. Mueller, Unitarity and the BFKL Pomeron, Nucl. Phys. B 437 (1995) 107 [hep-ph/9408245] [INSPIRE].

[10] Y.V. Kovchegov and E. Levin, Quantum chromodynamics at high energy, Cambridge Monographs on Particle Physics, Nuclear Physics and Cosmology, Cambridge University Press (2012) and references therein.

[11] I. Balitsky, Operator expansion for high-energy scattering, Nucl. Phys. B 463 (1996) 99 [hep-ph/9509348] [INSPIRE].

[12] I. Balitsky, Factorization and high-energy effective action, Phys. Rev. D 60 (1999) 014020 [hep-ph/9812311] [INSPIRE].

[13] Y.V. Kovchegov, Small x F(2) structure function of a nucleus including multiple Pomeron exchanges, Phys. Rev. D 60 (1999) 034008 [hep-ph/9901281] [INSPIRE].

[14] J. Jalilian-Marian, A. Kovner, A. Leonidov and H. Weigert, The BFKL equation from the Wilson renormalization group, Nucl. Phys. B 504 (1997) 415 [hep-ph/9701284] [INSPIRE]. 
[15] J. Jalilian-Marian, A. Kovner, A. Leonidov and H. Weigert, The Wilson renormalization group for low x physics: Towards the high density regime, Phys. Rev. D 59 (1998) 014014 [hep-ph/9706377] [INSPIRE].

[16] J. Jalilian-Marian, A. Kovner and H. Weigert, The Wilson renormalization group for low $x$ physics: Gluon evolution at finite parton density, Phys. Rev. D 59 (1998) 014015 [hep-ph/9709432] [INSPIRE].

[17] A. Kovner, J.G. Milhano and H. Weigert, Relating different approaches to nonlinear QCD evolution at finite gluon density, Phys. Rev. D 62 (2000) 114005 [hep-ph/0004014] [INSPIRE].

[18] E. Iancu, A. Leonidov and L.D. McLerran, The Renormalization group equation for the color glass condensate, Phys. Lett. B 510 (2001) 133 [hep-ph/0102009] [INSPIRE].

[19] E. Iancu, A. Leonidov and L.D. McLerran, Nonlinear gluon evolution in the color glass condensate. 1., Nucl. Phys. A 692 (2001) 583 [hep-ph/0011241] [INSPIRE].

[20] E. Ferreiro, E. Iancu, A. Leonidov and L. McLerran, Nonlinear gluon evolution in the color glass condensate. 2., Nucl. Phys. A 703 (2002) 489 [hep-ph/0109115] [INSPIRE].

[21] H. Weigert, Unitarity at small Bjorken x, Nucl. Phys. A 703 (2002) 823 [hep-ph/0004044] [INSPIRE].

[22] J.L. Albacete and C. Marquet, Gluon saturation and initial conditions for relativistic heavy ion collisions, Prog. Part. Nucl. Phys. 76 (2014) 1 [arXiv:1401.4866] [INSPIRE].

[23] E. Levin and K. Tuchin, Solution to the evolution equation for high parton density QCD, Nucl. Phys. B 573 (2000) 833 [hep-ph/9908317] [INSPIRE].

[24] A.H. Mueller and D.N. Triantafyllopoulos, The Energy dependence of the saturation momentum, Nucl. Phys. B 640 (2002) 331 [hep-ph/0205167] [INSPIRE].

[25] D.N. Triantafyllopoulos, The Energy dependence of the saturation momentum from $R G$ improved BFKL evolution, Nucl. Phys. B 648 (2003) 293 [hep-ph/0209121] [INSPIRE].

[26] E. Iancu, K. Itakura and L. McLerran, Geometric scaling above the saturation scale, Nucl. Phys. A 708 (2002) 327 [hep-ph/0203137] [INSPIRE].

[27] E. Iancu, K. Itakura and S. Munier, Saturation and BFKL dynamics in the HERA data at small x, Phys. Lett. B 590 (2004) 199 [hep-ph/0310338] [INSPIRE].

[28] S. Bondarenko, M. Kozlov and E. Levin, QCD saturation in the semiclassical approach, Nucl. Phys. A 727 (2003) 139 [hep-ph/0305150] [INSPIRE].

[29] J. Bartels and E. Levin, Solutions to the Gribov-Levin-Ryskin equation in the nonperturbative region, Nucl. Phys. B 387 (1992) 617 [InSPIRE].

[30] A.M. Stasto, K.J. Golec-Biernat and J. Kwiecinski, Geometric scaling for the total $\gamma^{*} p$ cross-section in the low $x$ region, Phys. Rev. Lett. 86 (2001) 596 [hep-ph/0007192] [INSPIRE].

[31] L. McLerran and M. Praszalowicz, Saturation and Scaling of Multiplicity, Mean $p_{T}$ and $p_{T}$ Distributions from $200 \mathrm{GeV}<\sqrt{s}<7 \mathrm{TeV}$ - Addendum, Acta Phys. Polon. B 42 (2011) 99 [arXiv: 1011.3403] [INSPIRE].

[32] L. McLerran and M. Praszalowicz, Saturation and Scaling of Multiplicity, Mean $p_{T}$ and $p_{T}$ Distributions from $200 \mathrm{GeV}<\sqrt{s}<7 \mathrm{TeV}$, Acta Phys. Polon. B 41 (2010) 1917 [arXiv: 1006.4293] [INSPIRE]. 
[33] M. Praszalowicz, Geometrical Scaling in Hadronic Collisions, Acta Phys. Polon. B 42 (2011) 1557 [arXiv: 1104.1777] [INSPIRE].

[34] M. Praszalowicz, Improved Geometrical Scaling at the LHC, Phys. Rev. Lett. 106 (2011) 142002 [arXiv: 1101.0585] [INSPIRE].

[35] E. Levin and K. Tuchin, Nonlinear evolution and saturation for heavy nuclei in DIS, Nucl. Phys. A 693 (2001) 787 [hep-ph/0101275] [INSPIRE].

[36] A. Kormilitzin, E. Levin and S. Tapia, Geometric scaling behavior of the scattering amplitude for DIS with nuclei, Nucl. Phys. A 872 (2011) 245 [arXiv:1106.3268] [INSPIRE].

[37] S. Munier and R.B. Peschanski, Traveling wave fronts and the transition to saturation, Phys. Rev. D 69 (2004) 034008 [hep-ph/0310357] [INSPIRE].

[38] S. Munier and R.B. Peschanski, Geometric scaling as traveling waves, Phys. Rev. Lett. 91 (2003) 232001 [hep-ph/0309177] [INSPIRE].

[39] E.A. Kuraev, L.N. Lipatov and V.S. Fadin, The Pomeranchuk Singularity in Nonabelian Gauge Theories, Sov. Phys. JETP 45 (1977) 199 [InSPIRE].

[40] Ya.Ya. Balitsky and L.N. Lipatov, The Pomeranchuk Singularity in Quantum Chromodynamics, Sov. J. Nucl. Phys. 28 (1978) 822 [Yad. Fiz. 28 (1978) 1597] [InSPIRE].

[41] Y.V. Kovchegov, Unitarization of the BFKL Pomeron on a nucleus, Phys. Rev. D 61 (2000) 074018 [hep-ph/9905214] [INSPIRE].

[42] I. Gradstein and I. Ryzhik, Tables of Series, Products, and Integrals fifth edition, Academic Press, London (1994).

[43] P. Hartman, Ordinary differential equations second edition, Birkhäuser, Boston-Basel-Stuttgart (1982).

[44] I.N. Sneddon, Elements of partial differential equations, Mc-Graw-Hill, New York (1957). 